\title{
¿Qué factores favorecen el conocimiento sobre los derechos de las personas con discapacidad?
}

\section{Gisvele Mena-Panclas ${ }^{1}$ Mercedes Llontop-Melendez ${ }^{1}$}

RESUMEN:

PALABRAS CLAVE

Citar como:
Objetivo: determinar los factores asociados al conocimiento sobre los derechos de las personas con discapacidad. Metodología: se realizó un análisis secundario de la Encuesta Nacional Especializada sobre Discapacidad 2012. En el análisis bivariado se empleó la prueba de Chi-cuadrado para la variables categóricas, mientras que en las numéricas la prueba de U de Mann-Whitney. Así mismo se utilizó la regresión de Poisson para calcular la Prevalencia de Ratio (Prevalence Ratio) con intervalos de confianza al 95\%. Resultados: los principales factores asociados al conocimiento sobre los derechos de las personas con discapacidad fueron: tener un nivel educativo alto (PR:10.20; IC 95\%:7.15-14.56), haber adquirido una enfermedad crónica (PR:1.18; IC 95\%:1.131.24), ser empleado (PR:1.83; IC 95\%:1.51-2.24) y estar registrado y tener un certificado en CONADIS (PR:4.87; IC 95\%:4.58-5.18). Sin embargo un factor que contribuye al desconocimiento fue tener discapacidad auditiva o intelectual (PR:0.65; IC 95\%: 0.60-0.70). Conclusión: tener un nivel educativo alto, ser empleado, poseer un seguro de salud y estar registrado en el CONADIS están asociados a conocer sobre los derechos de las personas con discapacidad. Mientras que tener una discapacidad auditiva o intelectual limita el conocimiento sobre estos derechos.

Personas con discapacidad; Conocimiento; Defensa de las personas con discapacidad.

Mena G, Llontop M. ¿Qué factores favorecen el conocimiento sobre los derechos de las personas con discapacidad? CASUS. 2017;2(3):147-155. 


\section{INTRODUCCIÓN}

La Organización Mundial de la Salud (OMS) define la discapacidad como una condición que tiene origen a partir de un elemento patológico en el ser humano (1). Como consecuencia se presenta una limitación en la función de sus capacidades físicas y mentales, afectando su participación en las actividades productivas (1). Sin embargo, las personas con alguna discapacidad no deberían encontrar en esta condición un motivo de limitación a su desarrollo y mucho menos un menor potenciamiento de su persona por el hecho de no conocer sus derechos; especialmente en relación a los derechos sobre su salud y no discriminación (2).

A nivel mundial mil millones de personas, lo que equivale a un $15 \%$ de la población general, padecen de algún tipo de discapacidad (3). En el Perú las personas con discapacidad son un millón quinientos setenta y cinco mil cuatrocientos dos personas lo que corresponde a un 5.2\% de la población nacional (4). Esta prevalencia aumentará debido a que la población tiende a alcanzar más años de vida (envejecimiento global) aumentando paralelamente su riesgo de discapacidad, lo cual se pudiera deber al aumento de enfermedades crónicas en este grupo (3). En respuesta a las múltiples necesidades se creó una Convención Internacional sobre los Derechos de las Personas con discapacidad, sin embargo muchas de estas personas no tienen acceso a los servicios de salud y de educación; presentando altas tasas de pobreza e índices bajos de salud. En consecuencia, esta problemática obstaculiza el acceso a otros sectores como el empleo y el transporte. Estas barreras están acompañadas, entre otros factores, por una ineficiente información sobre sus derechos (3).

Si bien la Convención Internacional de la ONU ha realizado ratificaciones para seguir beneficiando este sector de la población aún se siguen produciendo abusos y negligencias, puesto que estas se incumplen y no son puestas en práctica (5). En cuanto al conocimiento de sus derechos, el $33.7 \%$ de los peruanos con discapacidad los conoce, mientras que un $13.2 \%$ no lo conocen y solo un $30.6 \%$ conoce sobre la existencia de una ley o reglamento que favorece a las personas con discapacidad (6). El contexto donde se presentan barreras con mayor dificultad para ejercer sus derechos es en el trabajo debido a una falta de información detallada. Esto hace, por ejemplo, que los ciudadanos no puedan conocer los beneficios de contar con un seguro de salud (7). Cabe resaltar que respecto al conocimiento de los derechos, la discapacidad intelectual influye en el desconocimiento, dado que la persona puede presentar diferentes limitaciones en el funcionamiento mental y en sus destrezas cognitivas (8).

Se debe reconocer que a nivel nacional existe una carencia de servicios, políticas y programas de atención, orientación y apoyo a las personas con discapacidad lo que origina una falta de información en la población en general y en las personas con discapacidad (9). De igual manera, existe una falta de interés por parte de las autoridades sobre la integración de estas personas en la sociedad (10). Cabe resaltar que solo el $92.4 \%$ de las personas con discapacidad no cuentan con certificado de discapacidad (4). Por otro lado, existe un Consejo Nacional para la Integración de las Personas con Discapacidad (CONADIS), entidad que crea políticas, proyectos, programas, entre otros que favorecen la inclusión o la integración de las personas con discapacidad, pero lamentablemente un $89.0 \%$ de las personas con discapacidad no se encuentran registrados en la misma (4).

Ante esta problemática vinculada al desconocimiento de los derechos de las personas con discapacidad y la falta de estudios a nivel nacional sobre este fenómeno se propuso como objetivo: determinar los factores asociados al conocimiento sobre el derecho de las personas con discapacidad en el Perú, 2012. En tal sentido, el mismo pretende aportar información a los decisores de políticas públicas con el fin de que los ciudadanos con discapacidad puedan ejercer sus derechos y participar en programas de ayuda e inclusión social.

\section{MATERIALES Y MÉTODOS}

Para el análisis del estudio se utilizó la base de datos de la Encuesta Nacional Especializada sobre Discapacidad (ENEDIS) del año 2012. La misma fue brindada por el Instituto Nacional de Estadística e Informática (INEI) (3). La muestra efectiva estuvo conformada por 32528 personas con discapacidad. El muestreo fue probabilístico, estratificado, de áreas, bietápico y por conglomerados. Se incluyeron a todas las personas que vivían en zonas urbanas y rurales, de todos los departamentos del Perú. Se excluyeron a las personas menores de 18 años de edad.

La variable dependiente fue el conocimiento sobre los derechos de las personas con discapacidad, la misma 
se midió a través de las siguientes preguntas: ¿Conoce Ud. el consejo nacional para la integración de las personas (CONADIS)?, ¿Conoce Ud. la oficina regional de atención a la persona con discapacidad (OREDIS)?, ¿Conoce Ud. la oficina municipal de atención a la persona con discapacidad (OMAPED)?, ¿Conoce Ud. la defensoría del pueblo?, ¿Conoce Ud. organizaciones de personas con discapacidad?, ¿Conoce Ud. organizaciones pro defensa de los derechos humanos?, ¿Conoce Ud. organizaciones de rehabilitación?, ¿Conoce Ud. organizaciones religiosas de ayuda?, ¿Conoce Ud. alguna otra organización?, ¿No conoce Ud. ninguna organización?, ¿Conoce Ud. la convención de naciones unidas sobre los derechos de las personas con discapacidad?, ¿Conoce Ud. el convenio 159 de la organización internacional del trabajo (OIT) de la readaptación profesional de las personas con discapacidad?, ¿Conoce Ud. la ley general de la personas con discapacidad?, ¿Conoce Ud. la ley de atención preferente en lugares de atención al público?, ¿No Conoce Ud. ninguna de las normas descritas? Para el estudio, la variable se categorizó en sí conoce y no conoce.

Entre las variables sociodemográficas se consideró: edad: variable cuantitativa; número de hijos: variable cuantitativa; región variable categórica nominal (costa, sierra, selva); sexo variable categórica dicotómica (hombre, mujer); estado civil variable categórica politómica (soltero, separado, divorciado, viudo, casado, conviviente); nivel educativo variable politómica ordinal (sin nivel, inicial, primaria, secundaria, básica especial, técnico, universitario, maestría-doctorado); tipo de discapacidad variable categórica politómica (locomoción y destreza, visuales, voz y habla, auditivas, intelectual, conductarelaciones interpersonales); enfermedad crónica variable dicotómica (sí, no); seguro de salud variable dicotómica (sí, no), trabajo en la última semana variable dicotómica (sí, no); tipo de trabajo variable categórica (empleador, independiente, empleado: aquel que realiza actividades determinadas desarrollando habilidades intelectuales en una oficina que percibe pagos quincenales o mensuales, obrero: persona que realiza actividades operativas o manuales, generalmente recibe un pago por obra realizada, trabajar para un familiar sin remuneración, hogar, otro); relación sentimental variable dicotómica (sí, no); certificado de discapacidad variable dicotómica (sí, no) y registro en el CONADIS variable categórica (no, sí, no sabe).

En la realización del análisis estadístico se empleó el paquete estadístico STATA versión 12. En el análisis univariado se calcularon porcentajes para las variables categóricas, mientras que para las variables numéricas se calcularon medias y desviaciones estándares. Con respecto al análisis bivariado se utilizó la prueba de Chi-cuadrado para las variables categóricas y para las numéricas la prueba de U de Mann-Whitney. Se determinó la fuerza de asociación mediante la regresión de Poisson teniendo como resultados los Prevalence Ratio (PR) e intervalos de confianza al 95\% (IC 95\%). Los cuestionarios se realizaron de forma anónima por lo que no es posible identificar datos personales de los encuestados. La presente investigación fue presentada al Comité de Ética de Investigación de la Universidad Católica Sedes Sapientiae.

\section{RESULTADOS}

En la tabla 1 de las 32528 personas con discapacidad la edad media fue de 62 años, con un promedio de cinco hijos. El $48 \%$ pertenecía a la costa, el $53 \%$ de sexo femenino, el $35 \%$ se encontraba casado, el $42 \%$ contó con una educación primaria, el $62 \%$ contó con un seguro de salud, el $82 \%$ no trabajó la última semana, el 59\% tenía un trabajo independiente, el 93\% no contaba con el certificado de discapacidad, el $89 \%$ no estaba registrado en el CONADIS y el $81 \%$ no conocía sobre sus derechos.

Tabla 1. Características de las personas con discapacidad

\begin{tabular}{|c|c|c|}
\hline & $\mathbf{N}$ & $\%$ \\
\hline Edad m (DS) & $62.68(18.58)$ & \\
\hline Número de hijos m (DS) & $5.08(3.03)$ & \\
\hline \multicolumn{3}{|l|}{ Región } \\
\hline Costa & 15741 & 48.09 \\
\hline Sierra & 12285 & 37.53 \\
\hline Selva & 4706 & 14.38 \\
\hline \multicolumn{3}{|l|}{ Sexo } \\
\hline Mujer & 17373 & 53.08 \\
\hline Hombre & 15359 & 46.92 \\
\hline \multicolumn{3}{|l|}{ Estado civil } \\
\hline Soltero & 6213 & 18.98 \\
\hline Separado & 2518 & 7.69 \\
\hline Divorciado & 212 & 0.65 \\
\hline Viudo & 7802 & 23.84 \\
\hline Casado & 11758 & 35.92 \\
\hline Conviviente & 4229 & 12.92 \\
\hline
\end{tabular}


Tabla 1. continúa

\begin{tabular}{lcc}
\hline Nivel educativo & & \\
Sin nivel & 8059 & 24.62 \\
Inicial & 29 & 0.09 \\
Primaria & 13612 & 41.59 \\
Secundaria & 6947 & 21.22 \\
Básica especial & 371 & 1.13 \\
Técnico & 1688 & 5.16 \\
Universidad & 1984 & 6.06 \\
Maestría/doctor & 42 & 0.13
\end{tabular}

Tipo de discapacidad

$\begin{array}{lcc}\begin{array}{l}\text { Locomoción y } \\ \text { destreza }\end{array} & 6032 & 18.43 \\ \text { Visuales } & 7828 & 23.92 \\ \text { Voz y el habla } & 677 & 2.07 \\ \text { Auditivas } & 7159 & 21.87 \\ \text { Intelectual } & 5948 & 18.17 \\ \text { Conducta / } & 5088 & 15.54 \\ \text { Relaciones } & & \\ \text { Interpersonales } & & \\ \text { dad crónica } & & \\ \text { No } & 18571 & 56.74 \\ \text { Sí } & 14161 & 43.26\end{array}$

Seguro de salud

$\begin{array}{lll}\text { No } & 12557 & 38.36 \\ \text { Sí } & 20175 & 61.64\end{array}$

Trabajo en la última semana

No

Sí

\section{4 \\ 5928}

81.89

18.11

Tipo de trabajo

\begin{tabular}{lcc} 
Empleador & 387 & 5.39 \\
Independiente & 4287 & 59.53 \\
Empleado & 1043 & 14.52 \\
Obrero & 914 & 12.73 \\
Para familiar sin & & \\
remuneración & 422 & 5.88 \\
Hogar & 90 & 1.25 \\
Otro & 50 & 0.70 \\
sentimental & & \\
No & 4146 & 12.67 \\
Sí & 28586 & 87.33 \\
\hline
\end{tabular}

Conocer sobre los derechos de las personas con discapacidad se relaciona con tener una edad media inferior a 57 años, el haber alcanzado un nivel educativo de maestría o doctorado (76.19\%), tener un
Tabla 1. continúa

\begin{tabular}{ccc}
\hline $\begin{array}{l}\text { Certificado de } \\
\text { discapacidad } \\
\text { No }\end{array}$ Sí & 30.522 & \\
Registro en el CONADIS & 2210 & 6.75 \\
No & & \\
Sí & 29182 & 89.15 \\
No sabe & 1376 & 4.20 \\
Conocimiento sobre los & 2174 & 6.64 \\
derechos & & \\
No conoce & & \\
Si conoce & 26233 & 80.65 \\
& 6295 & 19.35 \\
\hline
\end{tabular}

trabajo dependiente (ser empleado) (56.86\%), poseer el certificado de discapacidad (65.89\%). Sin embargo, las personas que tienen una discapacidad auditiva o intelectual no conocen sobre sus derechos (ver tabla 2).

Tabla 2. Asociación entre el conocimiento sobre los derechos de las personas con discapacidad y los factores sociodemográficos

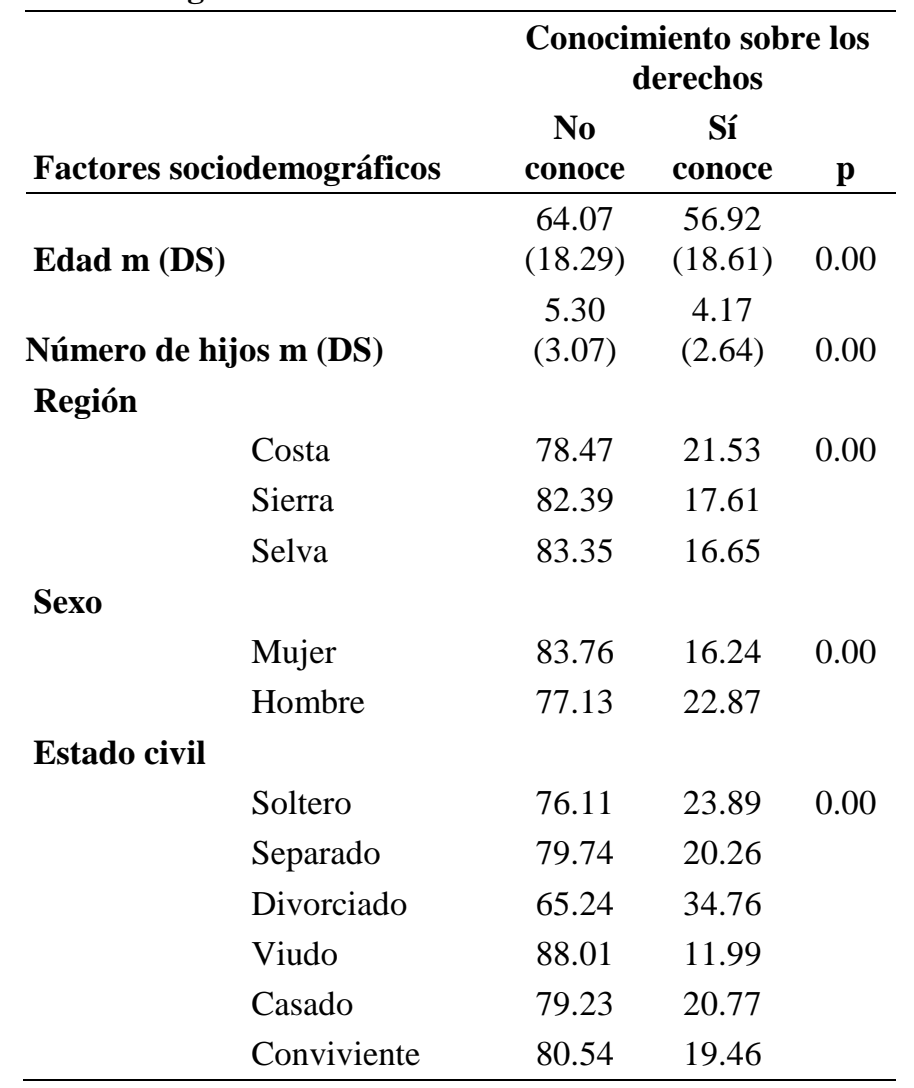


Tabla 2. continúa

\section{Nivel educativo}

Sin nivel

Inicial

Primaria

Secundaria

Básica especial

Técnico

Universidad

Maestría

doctor

Tipo de discapacidad

Locomoción y

destreza

Visuales

Voz y el habla

Auditivas

Intelectual

Conducta /

Relaciones

interpersonales

Enfermedad crónica

$\begin{array}{lll}\text { No } & 82.02 & 17.98 \\ \text { Sí } & 78.71 & 21.29\end{array}$

Seguro de salud

No

84.87

15.13

Sí

77.99

22.01

Trabajo en la última semana

$\begin{array}{lll}\text { No } & 82.55 & 17.45 \\ \text { Sí } & 71.42 & 25.58\end{array}$

Tipo de trabajo

\begin{tabular}{llll} 
Empleador & 69.03 & 30.97 & 0.00 \\
Independiente & 76.37 & 23.63 & \\
Empleado & 43.14 & 56.86 & \\
Obrero & 76.39 & 23.61 \\
Para familiar sin & & \\
remuneración & 84.00 & 16.00 \\
Hogar & 76.92 & 23.08 \\
Otro & 76.47 & 23.53 \\
\hline
\end{tabular}

Tabla 2. continúa

\begin{tabular}{cccc}
\hline Relación sentimental & & & \\
No & & & \\
Sí & 78.52 & 21.48 & 0.00 \\
Certificado de discapacidad & & & \\
No & 81.04 & 18.96 & \\
Sí & 84.17 & 15.83 & 0.00 \\
Registro en el CONADIS & 34.11 & 65.89 & \\
No & & & \\
Sí & 83.12 & 16.88 & 0.00 \\
No sabe & 89.72 & 10.28 & \\
\hline
\end{tabular}

En la tabla 3 se observa que los hombres tienen 0.39 veces más posibilidades de conocer sus derechos a comparación de las mujeres (PR:1.39; IC 95\%: 1.331.46), respecto al nivel educativo se muestra una mayor fuerza de asociación entre las personas con nivel educativo superior y los que conocen sus derechos en comparación a los otros niveles educativos inferiores (PR:10.20; IC 95\%: 7.15-14.56), 0.00 en las personas que tienen una discapacidad auditiva $\mathrm{o}$ intelectual en comparación con otro tipo de discapacidades disminuye la probabilidad de conocer sus derechos (PR:0.65; IC 95\%: 0.60- 0.70), también se evidencia que existe asociación entre una 0.00 enfermedad crónica y conocer sus derechos a diferencia de los que no tienen enfermedad crónica (PR:1.18; IC 95\%:1.13-1.24); respecto al ámbito laboral las personas que trabajaron la última semana se 0.00 asocia con conocer sus derechos en comparación a las personas que no trabajaron la última semana (PR 1.64 :IC 95\%: 1.54-1.73), también se observa asociación entre ser empleado y los que conocer sus derechos a diferencia de los otros tipos de trabajos (PR:1.83; IC $95 \%: 1.51-2.24)$. Se observa una mayor fuerza de asociación entre las personas con discapacidad que cuentan con certificado de discapacidad y los que conocen sobre sus derechos en comparación con los que no tienen certificado de discapacidad (PR: 4.16; IC 95\%: 3.93-4.40) y existe una mayor fuerza de asociación entre las personas con discapacidad que están registradas en el CONADIS y los que conocen sobre sus derechos en comparación con los que no están registrados (PR: 4.87; IC 95\%: 4.58-5.18). 
Tabla 3. Fuerza de asociación entre los conocimientos sobre los derechos de las personas con discapacidad y sus factores sociodemográficos

\begin{tabular}{llccc}
\hline & & \multicolumn{3}{c}{ Conocimiento sobre los } \\
derechos & PR & IC 95\% & p \\
\hline Edad & & 0.98 & $0.98-0.99$ & 0.00 \\
Número de hijos & 0.89 & $0.88-0.90$ & 0.00 \\
Región & & & & \\
& Costa & Ref & - & - \\
& Sierra & 0.81 & $0.77-0.85$ & 0.00 \\
& Selva & 0.75 & $0.69-0.81$ & 0.00 \\
Sexo & & & & \\
& Mujer & Ref & - & - \\
& Hombre & 1.39 & $1.33-1.46$ & 0.00 \\
Estado civil & & & \\
& Soltero & Ref & - & - \\
& Separado & 0.84 & $0.77-0.93$. & 0.00 \\
& Divorciado & 1.45 & $1.15-1.83$ & 0.00 \\
& Viudo & 0.50 & $0.46-0.54$ & 0.00 \\
& Casado & 0.87 & $0.82-0.92$ & 0.00 \\
& Conviviente & 0.81 & $0.75-0.88$ & 0.00
\end{tabular}

Nivel educativo

Sin nivel

Inicial

Primaria

Secundaria

Básica especial

Técnico

Universidad

Maestría doctor

Tipo de discapacidad

Locomoción y destreza

Visuales

Voz y el habla

Auditivas

Intelectual

Conducta /

Relaciones

interpersonales

Enfermedad crónica

\begin{tabular}{lccc} 
No & Ref & - & - \\
Sí & 1.18 & $1.13-1.24$ & 0.00 \\
\hline
\end{tabular}

Tabla 3. continúa

Seguro de salud

$\begin{array}{lccc}\text { No } & \text { Ref } & - & - \\ \text { Sí } & 1.45 & 1.38-1.53 & 0.00\end{array}$

Trabajo en la última semana

$\begin{array}{lccc}\text { No } & \text { Ref } & - & - \\ \text { Sí } & 1.64 & 1.54-1.73 & 0.00\end{array}$

Tipo de trabajo

$\begin{array}{lccc}\text { Empleador } & \text { Ref } & - & - \\ \text { Independiente } & 0.76 & 0.63-0.92 & 0.00 \\ \text { Empleado } & 1.83 & 1.51-2.24 & 0.00 \\ \text { Obrero } & 0.76 & 0.61-0.95 & 0.02 \\ \text { Para familiar sin } & & & \\ \text { remuneración } & 0.52 & 0.38-0.69 & 0.00 \\ \text { Hogar } & 0.74 & 0.47-1.18 & 0.21 \\ \text { Otro } & 0.76 & 0.42-1.38 & 0.36\end{array}$

Relación sentimental

$\begin{array}{lccc}\text { No } & \text { Ref } & - & - \\ \text { Sí } & 0.88 & 0.83-0.94 & 0.00\end{array}$

Certificado de discapacidad

$\begin{array}{lccc}\text { No } & \text { Ref } & - & - \\ \text { Sí } & 4.16 & 3.93-4.40 & 0.00\end{array}$

Registro en el CONADIS

\begin{tabular}{lccc} 
No & Ref & - & - \\
Sí & 4.87 & $4.58-5.18$ & 0.00 \\
No sabe & 0.61 & $0.53-0.69$ & 0.00 \\
\hline
\end{tabular}

\section{DISCUSIÓN}

En el estudio solo el $19.37 \%$ de los peruanos con discapacidad conocen sobre sus derechos. Además se observó una mayor fuerza de asociación en tener un mayor nivel educativo, ser empleado, presentar una enfermedad crónica, contar con el certificado de discapacidad y estar registrado en el CONADIS con un aumento del conocimiento sobre sus derechos en comparación con los que no conocen.

Un hallazgo importante del estudio fue que tener mayor nivel educativo se asocia con un aumento del conocimiento sobre los derechos de las personas con discapacidad (11). Esto se debe a que una persona con discapacidad con altos niveles de educación puede adquirir conocimientos sobre este tema debido al acceso de información a través de diferentes medios $(1,11,12)$. A diferencia de las personas que tienen una educación inferior, éstas no conocen siquiera que tienen derecho a una educación e igualdad de oportunidades (11). 
Del mismo modo, se evidenció que ser empleado se asoció positivamente con tener conocimientos sobre los derechos de las personas con discapacidad. Este hallazgo es similar en otro estudio (13). Lo anterior pudiera encontrar explicación en que este tipo de trabajo generalmente es formal y que la empresa brinde beneficios a sus empleados como el acceso a un control médico, prestaciones de ley, incentivos y liquidaciones (14). Esto hace que, por un lado, la persona ejerza una mayor responsabilidad en conocer más sobre sus derechos y, por el otro, se encuentren organizaciones que respaldan en caso de existir alguna irregularidad o maltrato por parte de la empresa.

Otro hallazgo importante fue que tener una enfermedad crónica se asoció con un conocimiento sobre sus derechos (15). Cuando una enfermedad es crónica indica que la persona lleva mucho tiempo conviviendo con ella. En este sentido, esto pudiera ser una motivación para buscar información sobre sus derechos y beneficios para ponerlo en práctica (16, 17). Asimismo, buscan tener un seguro de salud para adquirir los beneficios económicos que este tiene (17). Esto lo confirma un estudio que muestra que las personas con discapacidad que tienen un seguro de salud conocen más sobre sus derechos $(18,19)$. Debido a la aparición de los síntomas de una enfermedad crónica en las personas con discapacidad buscan conocer sus beneficios que le otorga el Estado para disminuir la carga económica y dependencia que pueden generar a sí mismos o a la familia (16).

Contar con el certificado de discapacidad y estar registrado en el CONADIS se asoció con tener conocimientos sobre los derechos de las personas con discapacidad (20). Esto se debe a que las personas que se registran en el CONADIS son más propensas a que ésta institución les mantenga informadas sobre las actualizaciones de las leyes y los derechos de las personas con discapacidad (21).

Finalmente, las personas con una discapacidad auditiva o intelectual tienen menos posibilidades de conocer sobre sus derechos $(22,23)$. Este último tipo de discapacidad genera dificultades y dependencia hacia los demás debido a que existe una limitación en el aprendizaje (5). Ya que el coeficiente intelectual de éstas personas, en muchos casos, es inferior a la media (24). De igual modo este problema pudiera estar conectado a que no han recibido una educación adecuada limitando el desarrollo de habilidades en la lectura y comprensión (25).

Las limitaciones del estudio se encuentran vinculadas a la recolección de los datos estadísticos que fueron obtenidos para un análisis primario. Por otra parte, las personas encuestadas no fueron clasificadas considerando su grado de dependencia, lo cual limitó el análisis e interpretación de los resultados. Entre las fortalezas se debe señalar que se contó con una muestra nacional representativa de las personas con discapacidad. Sin embargo, el conocimiento sobre los derechos de las personas con discapacidad puede estar asociado a otros factores que la ENEDIS no considero como por ejemplo de dondé o de quién recibió información sobre conocimiento sobre sus derechos. Asimismo, se recomienda mejorar el cuestionario de la ENEDIS principalmente en las preguntas en donde se indaga sobre el tipo de discapacidad que tiene la persona. En este sentido, el uso de la Clasificación Internacional del Funcionamiento, de la Discapacidad y de la Salud (CIE) es un instrumento adecuado de medición.

\section{CONCLUSIONES}

Los factores como tener un nivel educativo alto, ser empleado, poseer un seguro de salud, estar registrado en CONADIS y contar con un certificado de discapacidad están asociados al conocimiento sobre los derechos de las personas con discapacidad. Mientras que tener una discapacidad auditiva o intelectual limita el conocimiento sobre los derechos de las personas con discapacidad.

Los resultados de este estudio permiten identificar factores que favorecen o limitan el desarrollo de programas educativos por parte de profesionales o personas que brindan apoyo a las personas con discapacidad. Por esta razón, se recomienda que el Estado y las organizaciones relacionadas con las personas con discapacidad promuevan y mejoren el acceso a la información sobre sus derechos y que instauren nuevas estrategias. Así mismo, se debe dar prioridad a las políticas y servicios actuales creados para proteger y cumplir los derechos de las personas con discapacidad.

\section{REFERENCIAS BIBLIOGRÁFICAS}

1. Organización Mundial de la Salud. Discapacidades [internet]. Ginebra, Suiza: OMS; 2017 [citado el 1 de setiembre de 2017]. Disponible en: http://www.who.int/topics/disabilities/es/ 
2. Hernández A. Las personas con discapacidad: su calidad de vida y la de su entorno. Revista Aquichan, Bogotá 2004; 4(4).

3. Organización mundial de Salud. Informe mundial sobre la discapacidad [internet]. Ginebra, Suiza: OMS; 2011[citado el 1 de setiembre de 2017]. Disponible en: http://www.who.int/disabilities/world_report/2011/es/ 4. Instituto Nacional de Estadística e Informática. Primera encuesta nacional especializada sobre discapacidad 2012 [internet]. Lima, Perú: INEI; 2014 [citado el 1 de octubre de 2017]. Disponible en: https://www.inei.gob.pe/media/MenuRecursivo/public aciones_digitales/Est/Lib1171/ENEDIS\%202012\%20 -\%20COMPLETO.pdf

5. Gómez L, Verdugo $\mathrm{M}$, Arias $\mathrm{B}$, Irurtia $\mathrm{J}$. Evaluación de los derechos de las personas con discapacidad intelectual: estudio preliminar. Psicología Conductual. 2011;19(1):207-222.

6. Galarraga D, Mogro N. La aplicación de las medidas de protección vulneran los derechos de las personas con discapacidad en el Distrito Metropolitano de Quito, sector Carapungo Zona 2, en el año 2016 [Tesis de licenciatura]. Quito: Universidad Central del Ecuador; 2017.

7. Allaire SH, Evans SR, Lavalley MP, Merrigan DM. persons with rheumatic diseases and factors associated with use. Arthritis Care \& Research. 2001;45(2):174-182.

8. Centro Nacional de Diseminación de Información para Niños con Discapacidades. Discapacidades Intelectuales [internet]. Washington DC, Estado Unidos: NICHCY; 2010. Disponible en: https://www.parentcenterhub.org/wp-

content/uploads/repo_items/spanish/fs8sp.pdf

9. Pozos A, Hermosillo A. Conocimientos y opiniones de estudiantes de psicología sobre los derechos humanos de las personas con discapacidad. Revista Electrónica de Psicología Iztacala. 2015;18(4):14361456.

10. Salas B. Vulneración de los Derechos de las Personas con Discapacidad y Discriminación Social en la provincia de San Román- Juliaca 2013-2014 [Tesis de licenciatura]. Juliaca: Universidad Andina Néstor Cáceres Velásquez; 2015.

11.Barba J. Educación para los derechos humanos. 1a ed. México: Fondo de Cultura Económica; 2014.

12. Gentili, P. Marchas y contramarchas. El derecho a la educación y las dinámicas de exclusión incluyente en América Latina (a sesenta años de la Declaración Universal de los Derechos Humanos). Revista Iberoamericana de Educación. 2009;49(1):19-57.
13. Patlán J. Calidad de vida en el trabajo.1a ed. México: El Manual Moderno; 2017.

14. Villa N. Situación laboral de las personas con discapacidad en España. Revista Complutense de Educación. 2003;14(2):393-424.

15. Alvis N, Alvis L, Orozco J. Percepción sobre el derecho a la salud y acceso a servicios en usuarios del régimen subsidiado en un municipio colombiano, 2005. Revista de Salud Pública. 2008;10(3):386-394.

16. Álvarez LS. The right to health in Colombia: a proposal for laying down its moral foundations. Rev Panam Salud Publica. 2005;18(2):129-35.

17. Montiel L. Derecho a la salud en México. Un análisis desde el debate teórico contemporáneo de la justicia sanitaria. Revista IIDH. 2004;40(1):291-313.

18. Arbeláez M. Evaluación de la eficacia del derecho a la salud en Colombia a partir del proceso de descentralización sanitaria. Derecho y Salud. 2007;15(1):29-74.

19. Organización Panamericana de la Salud. Derechos humanos y derecho a la salud: construyendo ciudadanía en salud. Marco conceptual, aspecto metodológico y alcances operativos. Cuaderno de Promoción de la Salud. 2005;16:102.

20. Mosquera L, Hallo M. Data Mart para el sistema de servicios sociales del CONADIS. Revista Politécnica. 2014;33(1).

21. Consejo Nacional para la Integración de la Persona con Discapacidad. Marco legal [internet] Lima, Perú: CONADIS; 2017 [citado el 1 de octubre de 2017]. Disponible en: https://www.conadisperu.gob.pe/

22. Navas P, Gómez L, Verdugo M, Schalock R. Derechos de las personas con discapacidad intelectual: implicaciones de la convención de naciones unidas. Revista Española sobre Discapacidad Intelectual. 2012;43(3):7-28.

23. Storch de Gracia J. Derecho a la información y discapacidad (Una reflexión aplicada a los lenguajes de los sordos). Revista General de Información y Documentación. 2006;16(1):75-103.

24. Silverman W. Down syndrome: cognitive phenotype. Developmental Disabilities Research Reviews. 2007;13(3):228-236.

25. Van Gameren-Oosterom HB, Fekkes M, Buitendijk SE, Mohangoo AD, Bruil J, Van Wouwe JP. Development, problem behavior, and quality of life in a population based sample of eight-year-old children with Down syndrome. PLoS One. 2011;6(7):e21879. 
ISSN 2519-0652 CASUS.2017;2(3):147-155

\section{What factors favor knowledge about the rights of people with disabilities?}

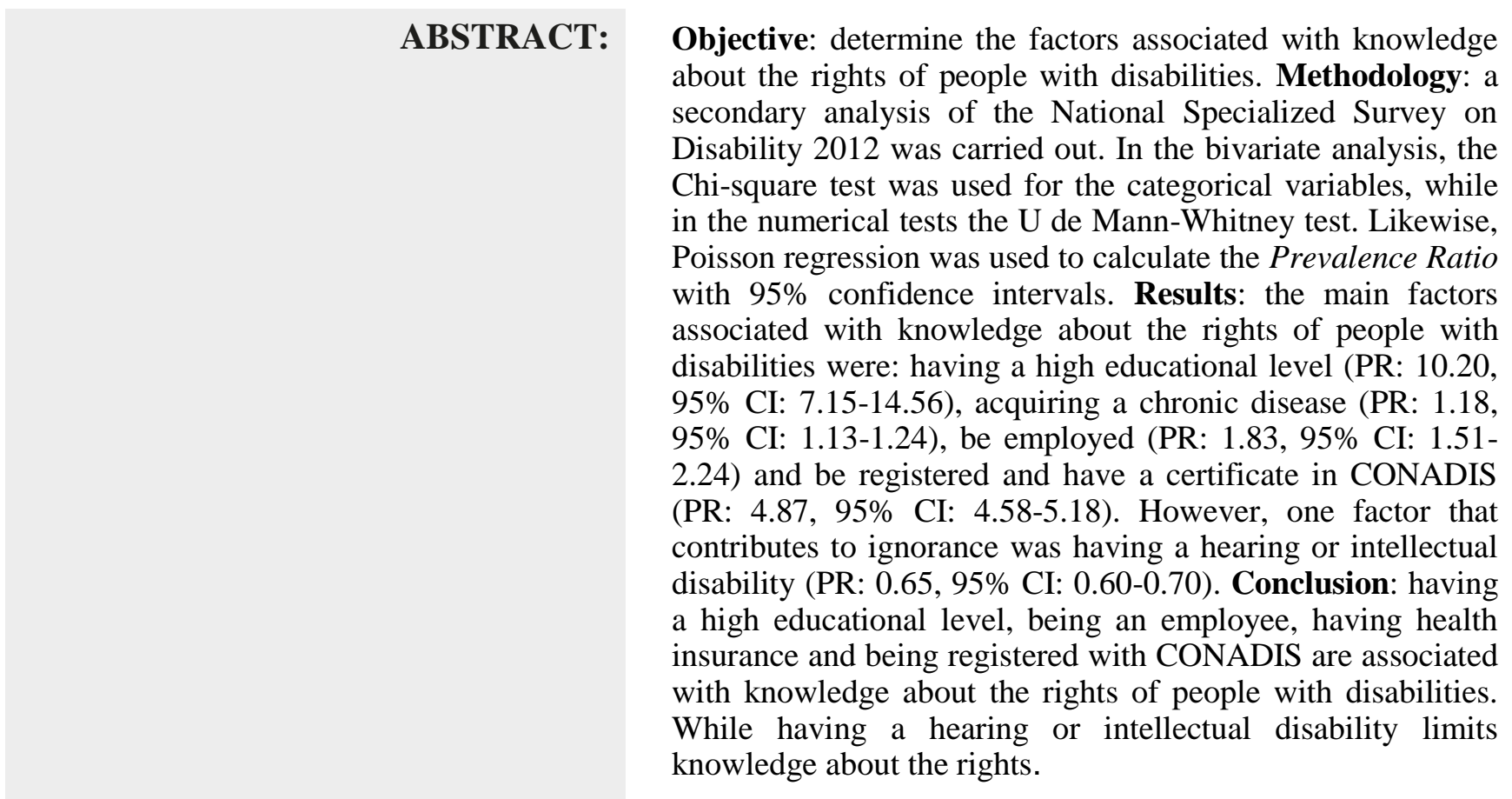

KEY WORDS: Disabled persons; Knowledge; Handicapped advocacy. 